\title{
Adaptação transcultural de um instrumento de avaliação do handicap auditivo para portadores de perda auditiva induzida pelo ruído ocupacional
}

\author{
Transcultural adaptation of an instrument to evaluate hearing \\ handicap in workers with noise induced hearing loss
}

\author{
Wanessa Tenório Gonçalves Holanda ${ }^{1}$ \\ M aria Luiza Carvalho de Lima ${ }^{2}$ \\ José $N$ atal Figueiroa ${ }^{3}$
}

${ }^{1}$ Subcoordenadoria de Vigilância Sanitária, Secretaria Estadual deSaúde Pública do Rio Grande do Norte. Av. JunqueiraAyres 488, Centro. 59025-280 Natal RN.

wanessa @hotmail.com

${ }^{2}$ Centro de Pesquisa Aggeu M agal hães, Fundação

Oswaldo Cruz.

${ }^{3}$ Instituto $M$ aterno Infantil

Professor Fernando

Figueira.
Abstract The noise induced hearing loss (NIHL) is a chronical and irreversible disease resulting of the exposure to noise in high levels at work. Even in the beginning, this hearing loss can damage in many degrees the worker's quality of life. Before this study, there wasn't an instrument, in Portuguese, to evaluate the psychosocial disadvantages of workers with NIHL. The aim of this research was to make a transcultural adaptation of an especific instrument to evaluate the hearing handicap from the original language to Portuguese, and check the reliability and legitimacy. The selected instrument passed by a process of semantic equivalence that was conducted in five stages: translation, back translation, critical appraisal of theversions, pretest and a final review by a multiprofessional group to develop a consensual version of the instrument for current use in Brazil. The instrument called "I nabilities Scale and $\mathrm{H}$ earing $\mathrm{H}$ andicap" had, in general, acceptable psychometrical measures, considering the little size of the sample and the fact that workers' hearing loss weren't too significant. Therefore, the Portuguese version of this instrument needs to be further tested in a representativesample of Brazilian workerswith N IH L to ratify its utility in order to evaluate hearing handicap in this population.

Key words Translation, Noise-induced hearing loss, Questionnaires
Resumo A Perda Auditiva Induzida pelo Ruído O cupacional (PAIRO) éuma doença crônica eirreversível resultante da exposição a elevados níveis de ruído no trabalho que, mesmo no estágio inicial, pode comprometer em vários graus a qualidade de vida do trabalhador. Não existia disponível para a língua portuguesa um instrumento específico de avaliação das implicações psicossociais para portadores de PAIRO. 0 objetivo desse trabalho foi realizar a adaptação transcultural de um instrumento de avaliação do handicap auditivo da língua original para o português e avaliar sua confiabilidade evalidade. 0 instrumento selecionado passou por um processo de equivalência semântica, que envolveu as etapas de tradução, retradução, apreciação formal da equivalência, pré teste e crítica final por uma equipe multiprofissional para a elaboração da versão final para uso corrente no Brasil. A Escala de Inabilidades e Handicap Auditivo obteve medidas psicométricas consideradas, em geral, aceitáveis, considerando o tamanho reduzido da amostra e o fato de os participantes não possuírem perdas auditivas muito acentuadas. Portanto, tal escala precisa ser testada em amostras maiores e representativas de trabaIhadores brasileiros portadores dePAIRO, a fim de ser ratificada a sua utilidade para a avaliação do handicap auditivo nessa população.

Palavras-chave Tradução, Perda auditiva induzida por ruído, Questionários 


\section{Introdução}

0 ruído é um som indesejável e, mais do que isso, ele representa um real perigo à saúde das pessoas ${ }^{1}$. 0 ruído constitui a maior causa isolada de risco ocupacional em todo o mundo edificilmente se verá um ambiente de trabalho totalmente desprovido desse risco².

A Perda Auditiva Induzida pelo Ruído O cupacional (PAIRO) é definida como uma doença crônica e irreversível resultante da agressão às células ciliadas do órgão deCorti, que decorreda exposição sistemática e prolongada a elevados níveis de ruído no ambiente de trabal ho ${ }^{3}$.

Pelo fato de a audição ter importante função social, a privação auditiva causa danos no comportamento individual, social epsíquico, influenciando na qualidade de vida dos seres humanos, podendo interferir na autoestima, na motivação e na eficácia no desenvolvimento do trabalho ${ }^{4}$.

Nesse sentido, a PAIRO deve ser encarada como uma potencial fonte de transtornos ao indivíduo acometido ${ }^{5}$, pois mesmo no seu estágio inicial, ela pode ser sentida e interferir na comunicação oral de seu portador, comprometendo em vários graus a qualidade de vida do mesmo ${ }^{6}$.

A PAIRO acometeinicial epredominantemente as frequências agudas e, por isso, os indivíduos portadores dessa perda de audição não vivem, necessariamente, num mundo silencioso. Muitos sons permanecem audíveis, mas são percebidos de forma distorcida e isso pode gerar uma inabilidade de conversar normalmente, tornando difícil a participação do trabalhador em encontros, reuniões, festas e outras manifestações públicas; além disso, atividades como ouvir televisão, rádio e telefone tornam-se difíceis ou impossíveis ${ }^{1}$.

A importância de sevalorizar a limitação psicossocial imposta pela PAIRO residenão somente na intenção de avaliar a saúde do trabalhador dentro do seu contexto global, mas também porque as repercussões psicossociais dessa doença podem se refletir a médio ou longo prazo no próprio desempenho profissional, originando ou agravando a incapacidade para o trabalho ${ }^{3}$.

A Organização M undial deSaúde ${ }^{7}$ definehandicap como sendo a desvantagem consequente deuma deficiência ou incapacidadequelimitaria ou impediria o indivíduo de desempenhar atividades consideradas normais para a idade, sexo, fatores culturais e sociais.

0 handicap auditivo poderia então ser considerado como a percepção, pelo indivíduo, de uma limitação na sua função psicossocial resultante da deficiência ou da incapacidade auditiva, que afetaria o seu estilo de vida, sua família, sua situação social e seu trabalho8.

Embora alguns autores afirmem que nem sempre a constatação de uma perda auditiva implica autopercepção do handicap auditivo ou que, pelo menos, as medidas audiométricas seriam insuficientes para descrever a reação do paciente em relação à sua perda auditiva e determinação da sua habilidade de comunicação na vida diária e na função psicossocial9-12, a associação entre a PAIRO e o handicap auditivo precisa ser melhor pesquisada através de estudos epidemiológicosque utilizem instrumentos demensuração válidos e que permitam a comparabilidade dos resultados encontrados.

No âmbito internacional, para quantificar as consequências psicossociais específicas da PAIRO, foram desenvolvidos al guns questionários, como por exemplo o Hearing M easurement Scale $(\mathrm{HMS})^{13}$, Hearing Handicap Scale $(\mathrm{HHS})^{14}$, Hearing $\mathrm{H}$ andicap and Support Scale(H HSS) ${ }^{15}$ eo He aring Disability and $\mathrm{H}$ andicap Scale (HDHS) ${ }^{16-18}$.

No Brasil, duas versões em português de instrumentos para avaliação do handicap auditivo são comumente utilizadas em pesquisas, sendo uma para idosos (H earing Handicap Inventory for Elderly - H HIE $)^{19}$ e outra para adultos (H earing $\mathrm{H}$ andicap Inventory for Adults - $\mathrm{HHIA}{ }^{10}$, não existindo nenhuma versão adaptada para 0 português de questionários específicos para trabalhadores portadores de PAIRO.

$N$ esse sentido, se faz urgente o desenvolvimento de métodos de avaliação dos impactos dessa perda auditiva, cuja prevalência na população de trabalhadores pode identificá-la como um dos mais importantes problemas ocupacionais em todo o mundo, especialmente nos países industrializados.

Esse estudo apresenta os resultados da adaptação transcultural de um instrumento de medida para avaliação do handicap auditivo em portadores de PAIRO para a língua portuguesa, com o objetivo de possibilitar e incentivar os pesquisadores brasileiros a estudar esse fenômeno no âmbito nacional, além de ampliar o conhecimento sobre métodos passíveis de serem adotados na área.

\section{Métodos}

A seleção do instrumento se deu a partir de uma pesquisa, utilizando como termos-chave "perda auditiva provocada por ruído", "handicap" e"questionários" nas bases de dados nacionais e inter- 
nacionais para, a partir da análise de cada instrumento utilizado, selecionar aquele que seria traduzido e adaptado para a língua portuguesa.

$\mathrm{O}$ instrumento selecionado foi o $\mathrm{H}$ earing $\mathrm{Di}$ sability and $\mathrm{H}$ andicap Scale (HDHS) ${ }^{16}$, que possui vinte questões e se apresenta como uma versão resumida do Hearing M easurement Scale ${ }^{13}$, primeiro instrumento de avaliação do handicap auditivo criado especificamente para a população de portadores de PAIRO.

Para a adaptação transcultural do instrumento, foi tomado como base o estudo de Reichenhein e $M$ oraes ${ }^{20}$, com as etapas de equivalência conceitual, de itens, semântica, operacional e de mensuração.

A equivalência conceitual foi realizada a partir de uma extensa pesquisa bibliográfica e análise de especialistas a respeito do conceito de handicap auditivo para a cultura brasileira e esse mesmo conceito em outras culturas.

A equivalência deitens teve como base a análisedeespecialistas para verificar seositens constantes no instrumento deveriam ser substituídos por não terem a mesma conotação da língua original.

A análise da equivalência semântica incluiu cinco etapas consecutivas: (1) tradução do instrumento original, (2) retradução, (3) apreciação formal de equivalência, (4) pré-teste com amostra de conveniência e (5) crítica final por especialistas na área, com elaboração da versão final. Cada uma dessas etapas será descrita a seguir.

$\mathrm{N}$ a etapa 1, foram realizadas duas traduções independentes do instrumento original (em inglês) para o português e ambas as traduções foram realizadas por fonoaudiólogos pós-graduados, fluentes na língua inglesa e conhecedores do objetivo da pesquisa. Ambos os tradutores possuíam certificado internacional de aprovação para as etapas de leitura e escrita na língua inglesa.

Buscou-se, no processo de tradução, respeitar a equivalência operacional, mantendo as características do instrumento original quanto ao número de itens, enunciado eopções de resposta.

A etapa 2 consistiu na retradução das duas versões em português por outros dois tradutores bilíngues independentes, sendo um fonoaudiólogo e outro profissional de outra área de conhecimento. Ambos possuíam certificado internacional de aprovação nas habilidades de leitura e escrita e não conheciam os objetivos do estudo. As retraduções foram cegas em relação ao perfil dos profissionais da primeira etapa.

A terceira etapa teve como objetivo a apreciação formal de equivalência semântica a partir do julgamento dos itens quanto ao significado refe- rencial e geral dos termos e das expressões constantes nos mesmos.

0 significado referencial diz respeito à correspondência literal que existe entre as palavras do instrumento original edas retraduções ${ }^{21}$. Nessa fase da equivalência semântica, procurou-se comparar em cada questão retraduzida a sua equivalência com a mesma questão constante no instrumento original, buscando classificar essa equivalência em porcentagens de 0 a 100\%, sendo $0 \%$ a completa discrepância literal dos termos e $100 \%$ a completa equivalência dos mesmos. Essa etapa foi denominada de A1.

Foram realizadas duas avaliações para o significado referencial das questões, sendo ambas feitas por professores de inglês (língua original do questionário), um tendo como língua nativa o inglês e o outro, o português. Vale ressaltar que ambos apresentavam domínio completo da língua portuguesa e as avaliações foram realizadas de forma independente.

O significado geral leva em conta aspectos mais sutis que a simples correspondência literal dos termos, objetivando verificar a representação das ideias (conceitos) a que uma única palavra ou conjunto de palavras aludem ${ }^{21}$.

Para o significado geral, optou-se por selecionar indivíduos bilíngues com amplo conhecimento teórico e prático na área de fonoaudiologia para a apreciação dos pares de itens. Três profissionais realizaram de forma independente cada avaliação e, a partir dessas análises, foram selecionadas as questões para compor a versão em português do Hearing Disability and Handicap Scale.

Foram usados formulários específicos para cada aspecto de equivalência semântica. Para o significado referencial, foi utilizado um formulário que continha pares de itens do questionário original em inglês com as duas retraduções realizadas, de forma que seriam comparados os seguintes pares: original com retradução 1 , original com retradução 2 e retradução 1 com retradução 2. Esta última comparação não foi avaliada formalmente e foi incluída apenas com o objetivo de evitar que os avaliadores identificassem a origem das questões.

Para a avaliação do significado geral, optouse por uma classificação qualitativa em quatro categorias: inalterado (IN), pouco alterado (PA), muito alterado ( $\mathrm{MA}$ ) e completamente alterado (CA). Eram apresentados no formulário os seguintes pares de itens: original com tradução 1 e original com tradução 2. Dessa forma, o avaliador tinha conhecimento da pergunta original, 
avaliando o seu significado geral eo impacto produzido na população de origem e comparava com cada tradução realizada, sendo orientado a enquadrar os pares de questões dentre as quatro possibilidades de classificação. Essa etapa foi denominada de $\mathrm{A} 2$.

Para a versão final, al guns termos foram incorporados de uma ou das duas versões e, em alguns casos, optou-se por certas modificações no intuito de tornar as perguntas mais fáceis de serem compreendidas por indivíduos numa faixa ampla de escolaridade, sempre com o cuidado de preservar o sentido de cada questão.

A etapa 4 consistiu na aplicação da versãosíntese em uma amostra de conveniência composta de dez trabalhadores expostos ao ruído ocupacional. Os participantes do pré-teste trabal havam em uma indústria de bebidas da cidade do Recifee no setor de lavanderia de um hospital universitário localizado na mesma cidade.

A aplicação da versão-síntese teve como finalidade principal analisar a compreensão dos termosutilizados com o propósito de verificar possíveis ajustes e, por isso, justifica-se o critério de seleção de uma amostra por conveniência, visto que 0 objetivo não era a representatividade da amostra, mas o aprimoramento do questionário.

N essa etapa, solicitava-se do entrevistado que classificassea pergunta como defácil ou dedifícil entendimento e que desse um exemplo de sua vida diária correspondente ao item que acabara de responder. 0 entrevistador, então, julgava a compreensão do entrevistado com base na pertinência do exemplo, registrando os comentários para cada item. A aplicação do questionário foi realizada pela pesquisadora responsável edurou, em média, trinta minutos.

A compreensão do entrevistado era classificada em enten dimento correto ou entendimento incorreto/duvidoso. As questões que não alcançassem entendimento correto ou fossem classificadas como de difícil entendimento por mais de $10 \%$ dos entrevistados deveriam ser reajustadas. Nesses casos, era solicitado do entrevistado que sugerisse uma melhor forma de formular a questão para que essa elaboração da pergunta sugerida fosse avaliada posteriormente.

A etapa 5 objetivou discutir e analisar os comentários registradossobreositens com uma equipe multiprofissional, a fim de adequar a versãosíntese e orientar a elaboração da versão final.

A equipe multiprofissional foi composta por dois fonoaudiólogos com experiência na área de saúde ocupacional; um médico do trabalho, epidemiologista; uma professora de português, com
23 anos de experiência; e um voluntário denível médio.

A equivalência operacional foi mantida através do mesmo número de questões da versão original, mesmas opções de resposta e método de aplicação do questionário (autoadministrado) ea equivalência de mensuração foi realizada a partir da avaliação da confiabilidade evalidade da versão final.

A versão final foi elaborada eaplicada, juntamente com um questionário de caracterização da amostra, por um único entrevistador (pesquisadora responsável) em trabalhadores portadores de PAIRO de uma indústria de bebidas da cidade do Recife. Essa indústria possuía um total de 175 trabalhadores expostos ao ruído ocupacional; dentre estes, um total de 48 trabaIhadores portadores de PAIRO em algum grau. Todos os trabalhadores com PAIRO foram convidados a participar desta pesquisa, porém cinco deles não puderam ser incluídos, pois dois encontravam-se de férias e três estavam afastados do trabalho por motivos de saúde no período de coleta de dados.

Os trabalhadores portadores de PAIRO participantes da pesquisa $(n=43)$ responderam individual mente à Escala de Inabilidades e H andicap Auditivo e foram retiradas, de seu prontuário, informações a respeito do último exame audiométrico realizado.

Todos os dados referentes ao exame audiométrico e ao preenchimento da escala foram armazenados e analisados através dos programas Epi Info versão 3.3.2 eSPSS versão 13.

Para avaliar a confiabilidade e validade da versão final obtida após o processo de adaptação transcultural, inicialmente foi realizada a estatística descritiva das respostas aos itens do questionário, seguida de análise fatorial, correlações item-total e análise da consistência interna do instrumento (pelo alfa de Cronbach).

A confiabilidade é definida como o grau de precisão ou de coerência com que o instrumento mede 0 atributo que se propõe a medir e o coeficientealfa medea intercorrelação dositens eproporciona uma estimativa do erro de medida ${ }^{22}$.

Para a análise fatorial, foi realizada uma análise de componente principal (ACP) seguida de rotação varimax nos vinte itens da versão adaptada para a língua portuguesa. 0 "Cattel scree test" foi utilizado como critério para extração dos fatores. Não foi realizada uma análise confirmatória, tendo em vista que o tamanho amostral não era suficientemente grande para produzir estimativas estáveis dos parâmetros do modelo. 
Todos os trabalhadores assinaram um termo de consentimento livre eesclarecido, no qual ficaram cientes dos objetivos da pesquisa e de sua importância. Os nomes dos participantes não foram divulgados e essa pesquisa foi aprovada pelo Comitê de Ética em Pesquisa do Centro de Ciências da Saúde da UniversidadeFederal de Pernambuco.

\section{Resultados}

A análise da equivalência conceitual demonstrou que o conceito de handicap auditivo para a cultura brasileira e para outras culturas foi considerado equivalente. Além disso, não foram identificados itens que deveriam ser substituídos por não terem a mesma conotação do termo original, seguindo-se então para a etapa de equivalência semântica.

$\mathrm{Na}$ avaliação da versão com maior correspondêncialiteral, identificou-se que os avaliadores apresentaram uma concordância de $85 \%$ e isso demonstra que, de forma geral, o julgamento dos avaliadores foi semelhante. Os percentuais médios de avaliação da correspondência literal (A1) variaram de 65 a $100 \%$.

$\mathrm{Na}$ fase de análise do significado geral (A2), os examinadores foram solicitados a comparar os itens traduzidos e a versão original, verificando a equivalência de forma qualitativa em quatro categorias: inalterado (IN), pouco alterado (PA), muito alterado (MA) e completamente alterado (CA).

Para a grande maioria das avaliações realizadas, os profissionais consideraram os itens traduzidos como estando inalterados, comparados à versão original em inglês.

Alguns termos foram incorporados de uma ou das duas versões e, em al guns casos, optou-se por certas modificações com o objetivo de tornar as perguntas mais fáceis de serem compre endidas por indivíduos numa faixa ampla de escolaridade, sempre com o cuidado de preservar o sentido de cada questão. A sinopse do processo de decisão para a criação da versão-síntese encontra-se disposta na Tabela 1.

No pré-teste, as perguntas foram julgadas como de fácil entendimento pelos entrevistados e todas elas foram compreendidas corretamente por $90 \%$ ou mais dos entrevistados. Apenas três perguntas necessitaram de modificações por terem sido julgadas por $20 \%$ da população como de difícil entendimento (questões 4, 8 e 20).
Outra dúvida bastante frequente dos participantes do pré-teste estava relacionada às opções de resposta. Muitos tiveram dificuldades na marcação das respostas e sugeriram a colocação da palavra "sim" ou "não" antes de cada alternativa, ficando as opções com a seguinte composição: "Não, nunca"; "Sim, algumas vezes"; "Sim, frequentemente"; e "Sim, sempre". Essas modificações foram aceitas, visto que não modificam as alternativas no seu sentido principal, apenas incluem palavras que facilitam o entendimento e marcação da resposta correta.

$\mathrm{Na}$ Tabela 2, são apresentados os resultados das etapas de tradução, retradução e avaliação dos significados referencial (A1) e geral (A2).

O Gráfico 1 apresenta os limiares auditivos médios de cada frequência (de $500 \mathrm{a} 8000 \mathrm{~Hz}$ ) para as orelhas direita e esquerda. Percebe-se que os trabalhadores participantes da pesquisa possuíram, em média, limiares auditivos dentro do padrão de normalidade (até 25dB NA) para as bai xas frequências e limiares auditivos alterados para a grande maioria das altas frequências (grau leve), para ambas as orelhas.

Tabela 1. Sinopse do processo de decisão relativo à criação da versão-síntese do questionário traduzido e adaptado transculturalmente.

\begin{tabular}{|c|c|c|c|c|c|}
\hline Item & V 1 & & V2 & $\mathrm{V} 1+\mathrm{V} 2$ & Modificado \\
\hline 1 & $\checkmark$ & $\sim$ & $\checkmark$ & & \\
\hline 2 & $\checkmark$ & $\sim$ & $\checkmark$ & & \\
\hline 3 & $\checkmark$ & $\sim$ & $\checkmark$ & & \\
\hline 4 & $\checkmark$ & $=$ & $\checkmark$ & & \\
\hline 5 & $\checkmark$ & $\sim$ & $\checkmark$ & & \\
\hline 6 & $\checkmark$ & $\sim$ & $\checkmark$ & & \\
\hline 7 & $\checkmark$ & $\Rightarrow$ & $\checkmark$ & & \\
\hline 8 & $\checkmark$ & $=$ & $\checkmark$ & & \\
\hline 9 & $\checkmark$ & $\sim$ & $\checkmark$ & & \\
\hline 10 & $\checkmark$ & $\sim$ & $\checkmark$ & $\rightarrow$ & $\checkmark$ \\
\hline 11 & $\checkmark$ & $\sim$ & $\checkmark$ & & \\
\hline 12 & $\checkmark$ & $\sim$ & $\checkmark$ & & \\
\hline 13 & $\checkmark$ & $\sim$ & $\checkmark$ & & \\
\hline 14 & $\checkmark$ & $=$ & $\checkmark$ & & \\
\hline 15 & $\checkmark$ & $\sim$ & $\checkmark$ & & \\
\hline 16 & $\checkmark$ & $\sim$ & $\checkmark$ & & \\
\hline 17 & $\checkmark$ & $\sim$ & $\checkmark$ & & \\
\hline 18 & $\checkmark$ & $\sim$ & $\checkmark$ & & \\
\hline 19 & $\checkmark$ & $\sim$ & $\checkmark$ & & \\
\hline 20 & $\checkmark$ & $\sim$ & $\checkmark$ & & \\
\hline
\end{tabular}

$\checkmark$ indica a escolha da versão-síntese.

$\sim$ indica semelhança entre os itens nas duas versões.

= indica que as duas versões foram idênticas.

$\mp$ indica que as duas versões foram diferentes. 
Tabela 2. Avaliação da equivalência semântica entre a versão original em inglês do instrumento Hearing Disability and Handicap Scale e suas versões em português.

\begin{tabular}{|c|c|c|c|c|c|c|}
\hline \multirow[t]{2}{*}{ Formulário original } & \multirow[t]{2}{*}{ Traduções } & \multirow[t]{2}{*}{ Retraduções } & \multirow{2}{*}{$\begin{array}{l}\text { Al- } \\
\text { Significado } \\
\text { referencial }\end{array}$} & \multicolumn{3}{|c|}{ A2- Significado geral } \\
\hline & & & & Exam 1 & Exam 2 & Exam 3 \\
\hline \multirow[t]{2}{*}{$\begin{array}{l}\text { 1) Do you have difficulty } \\
\text { following a conversation } \\
\text { normally in any of the } \\
\text { following situations: at } \\
\text { work, in a bus or a car, or } \\
\text { when shopping? }\end{array}$} & $\begin{array}{l}\text { T1 Você tem dificuldade } \\
\text { de acompanhar uma } \\
\text { conversação } \\
\text { normalmente em } \\
\text { alguma dessas } \\
\text { situações: no trabalho, } \\
\text { no ônibus ou no carro } \\
\text { ou quando vai às } \\
\text { compras? }\end{array}$ & $\begin{array}{l}\text { R1D o you have any } \\
\text { difficult to follow a } \\
\text { conversation normally } \\
\text { in any of these } \\
\text { situations: at work, in } \\
\text { the bus or in the car or } \\
\text { shopping? }\end{array}$ & $95 \%$ & PA & IN & IN \\
\hline & $\begin{array}{l}\text { T2 Você tem dificuldade } \\
\text { de manter uma } \\
\text { conversação normal } \\
\text { em alguma dessas } \\
\text { situações: no trabalho, } \\
\text { no ônibus ou no carro } \\
\text { ou quando está } \\
\text { comprando? }\end{array}$ & $\begin{array}{l}\text { R2 D o you have difficulty } \\
\text { in maintaining a } \\
\text { normal conversation } \\
\text { in some of those } \\
\text { situations: at work, on } \\
\text { the bus or in the car or } \\
\text { when shopping? }\end{array}$ & $65 \%$ & IN & PA & PA \\
\hline \multirow[t]{2}{*}{$\begin{array}{l}\text { 2) Can you hear the } \\
\text { sound of the door } \\
\text { opening when you're } \\
\text { inside the room? }\end{array}$} & $\begin{array}{l}\text { T1 Você pode escutar o } \\
\text { som de uma porta } \\
\text { abrindo quando você } \\
\text { está dentro da sala? }\end{array}$ & $\begin{array}{l}\text { R1 Could you hear the } \\
\text { sound of a door } \\
\text { opening when you are } \\
\text { inside of the room? }\end{array}$ & $95 \%$ & IN & IN & IN \\
\hline & $\begin{array}{l}\text { T2 Você consegue escutar } \\
\text { o som de uma porta } \\
\text { abrindo quando você } \\
\text { está dentro do quarto? }\end{array}$ & $\begin{array}{l}\text { R2 Can you listen the } \\
\text { sound of a door } \\
\text { opening when you are } \\
\text { inside of the room? }\end{array}$ & $80 \%$ & IN & IN & IN \\
\hline \multirow[t]{2}{*}{$\begin{array}{l}\text { 3) Do you worry that } \\
\text { people will find out you } \\
\text { have a hearing problem? }\end{array}$} & $\begin{array}{l}\text { T1 Você se importa que as } \\
\text { pessoas descubram que } \\
\text { você tem um } \\
\text { problema de audição? }\end{array}$ & $\begin{array}{l}\text { R1Do you mind that } \\
\text { people find out that } \\
\text { you have a hearing } \\
\text { problem? }\end{array}$ & $75 \%$ & PA & PA & PA \\
\hline & $\begin{array}{l}\text { T2 Você se preocupa } \\
\text { (sentido negativo da } \\
\text { palavra) queas pessoas } \\
\text { descubram que você } \\
\text { tem um problema } \\
\text { auditivo? }\end{array}$ & $\begin{array}{l}\text { R2 Do you mind that the } \\
\text { people find out you } \\
\text { have a hearing } \\
\text { problem? }\end{array}$ & $90 \%$ & IN & IN & IN \\
\hline \multirow[t]{2}{*}{$\begin{array}{l}\text { 4) Is it difficult for you } \\
\text { to ask people to repeat } \\
\text { themselves? }\end{array}$} & $\begin{array}{l}\text { T1 É difícil para você } \\
\text { pedir que as pessoas } \\
\text { repitam? }\end{array}$ & $\begin{array}{l}\text { R1 Is it hard to you asking } \\
\text { people to repeat? }\end{array}$ & $70 \%$ & IN & IN & IN \\
\hline & $\begin{array}{l}\text { T2 É difícil para você } \\
\text { pedir que as pessoas } \\
\text { repitam? }\end{array}$ & $\begin{array}{l}\mathrm{R} 2 \text { Is it difficult for you to } \\
\text { ask the people to } \\
\text { repeat? }\end{array}$ & $100 \%$ & IN & IN & IN \\
\hline \multirow[t]{2}{*}{$\begin{array}{l}\text { 5) Do you have difficulty } \\
\text { hearing what's being said } \\
\text { on TV if someone other } \\
\text { than yourself adjusts the } \\
\text { volume? }\end{array}$} & $\begin{array}{l}\text { T1 Você tem dificuldade } \\
\text { de escutar o que está } \\
\text { sendo dito na TV se } \\
\text { outra pessoa ajustar o } \\
\text { volume? }\end{array}$ & $\begin{array}{l}\text { R1Do you have any } \\
\text { difficult to hear what } \\
\text { has been said on TV if } \\
\text { someone else adjusts } \\
\text { the volume? }\end{array}$ & $80 \%$ & PA & IN & IN \\
\hline & $\begin{array}{l}\text { T2 Você tem dificuldade } \\
\text { de ouvir o que está } \\
\text { sendo dito na TV se } \\
\text { alguém fora você } \\
\text { mesmo ajustar o } \\
\text { volume? }\end{array}$ & $\begin{array}{l}\text { R2 Do you have difficulty } \\
\text { of hearing what is } \\
\text { being said on tv if } \\
\text { anybody else adjust } \\
\text { the volume? }\end{array}$ & $95 \%$ & IN & IN & IN \\
\hline
\end{tabular}


Tabela 2. continuação

Formulário original

Traduções

Retraduções

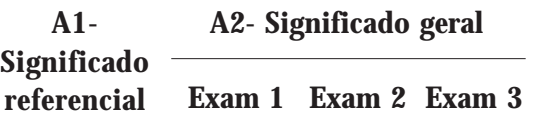

6) Can you hear the water boiling in the pan when you're in the kitchen?

7) Do you get upset if you give the wrong answer to someone because you've misheard them?

8) Does your hearing condition restrict your social or personal life?

9) Do you have difficulty hearing what's being said on the radio if someone other than yourself adjusts the volume?

10) Can you hear the footsteps of someone coming into the room without you seeing them?

11) Does it bother or upset you if you are unable to follow a conversation?
T1 Você consegue ouvir a R1Could you hear the água fervendo na panela quando você está na cozinha?

T2 Você consegue escutar a água fervendo na panela quando você está na cozinha?

T1 Você fica chateado se dá uma resposta errada a alguém porque você não a escutou?

T2 Você fica contrariado se você dá uma resposta errada a alguém porque você escutou mal?

T1 A sua condição auditiva restringe a sua vida social ou pessoal?

T2 A sua condição auditiva restringe a sua vida social ou pessoal?

T1 Você tem dificuldade de escutar o que está sendo dito na rádio se outra pessoa ajustar o volume?

T2 Você tem dificuldade de ouvir o que está sendo dito no rádio se alguém fora você mesmo ajustar o volume?

T1 Você pode ouvir os passos de al guém entrando na sala sem você vê-la? water boiling in the pan when you are in the kitchen?

R2Can you listen to the water boiling in the pot when you are at the kitchen?

R1Do you get upset if you give a wrong answer to someone because you didn't hear?

R2Are you disappointed if you give a wrong answer to anybody because you listened badly?

R1D oes your hearing condition restrict your social/personal life?

R2D oes your hearing condition restrict your social life or personal?

R1D o you have any difficult to hear what has been said in the radio if someone else adjusts the volume?

R2D o you have difficulty of hearing what is being said in the radio if anybody else adjust the volume?

R1Could you hear someone's steps walking through the room without seeing?

T2 Você consegue escutar R2 Can you listen the os passos de al guém entrando da sala sem que você o veja?

T1 Você se sente incomodado ou chateado se você não consegue acompanhar uma conversação?

T2 Incomoda ou constrange o fato de não conseguir manter uma conversação? steps of anybody entering of the room without you see it?

R1Did bother or upset you if you aren't able to follow a conversation?

R2Does it inconvenience or constrain the fact of not getting to maintain a conversation?

Exam 1 Exam 2 Exam 3

$95 \% \quad$ IN IN IN

$80 \% \quad$ PA IN IN

$80 \% \quad M A \quad M A \quad M A$

$80 \% \quad$ IN IN IN IN

$\begin{array}{llll}100 \% & \text { IN } & \text { IN } & \text { IN } \\ 90 \% & \text { IN } & \text { IN } & \text { IN } \\ 95 \% & \text { PA } & \text { IN } & \text { IN }\end{array}$

$\begin{array}{lll}85 \% & \text { IN } & \text { IN }\end{array}$




\begin{tabular}{|c|c|c|c|c|c|c|}
\hline Tabela 2. continuação & & & & & & \\
\hline \multirow[t]{2}{*}{ Formulário original } & \multirow[t]{2}{*}{ Traduções } & \multirow[t]{2}{*}{ Retraduções } & \multirow{2}{*}{$\begin{array}{l}\text { A1- } \\
\text { Significado } \\
\text { referencial }\end{array}$} & \multicolumn{3}{|c|}{ A2- Significado geral } \\
\hline & & & & Exam 1 & Exam 2 & Exam 3 \\
\hline \multirow[t]{2}{*}{$\begin{array}{l}\text { 12) Do you find that you } \\
\text { are more tense and tired } \\
\text { because of your hearing } \\
\text { difficulty? }\end{array}$} & $\begin{array}{l}\text { T1 Você acha que você } \\
\text { está mais tenso e } \\
\text { cansado por causa da } \\
\text { sua dificuldade de } \\
\text { ouvir? }\end{array}$ & $\begin{array}{l}\text { R1 Do you think that you } \\
\text { are more stressed or } \\
\text { tiredness because of } \\
\text { the hearing difficulty? }\end{array}$ & $85 \%$ & IN & PA & IN \\
\hline & $\begin{array}{l}\text { T2 Você acha que você } \\
\text { está mais tenso e } \\
\text { cansado por causa da } \\
\text { sua dificuldade } \\
\text { auditiva? }\end{array}$ & $\begin{array}{l}\text { R2 Do you think you have } \\
\text { more tension or are } \\
\text { more tired because of } \\
\text { your hearing difficulty? }\end{array}$ & $85 \%$ & IN & IN & IN \\
\hline \multirow[t]{2}{*}{$\begin{array}{l}\text { 13) Do you have } \\
\text { difficulty hearing in } \\
\text { group conversations? }\end{array}$} & $\begin{array}{l}\text { T1 Você tem dificuldade } \\
\text { de ouvir em conversas } \\
\text { em grupo? }\end{array}$ & $\begin{array}{l}\text { R1 Do you have any } \\
\text { difficult to hear a } \\
\text { group's conversations? }\end{array}$ & $90 \%$ & IN & IN & IN \\
\hline & $\begin{array}{l}\text { T2 Você tem dificuldade } \\
\text { auditiva em conversas } \\
\text { em grupo? }\end{array}$ & $\begin{array}{l}\text { R2 Do you have hearing } \\
\text { difficulty in a group } \\
\text { conversations? }\end{array}$ & $90 \%$ & IN & IN & IN \\
\hline \multirow[t]{2}{*}{$\begin{array}{l}\text { 14) Do you hear when } \\
\text { someone rings the } \\
\text { doorbell or knocks the } \\
\text { door? }\end{array}$} & $\begin{array}{l}\text { T1 Você escuta quando } \\
\text { alguém toca a } \\
\text { campainha ou bate na } \\
\text { porta? }\end{array}$ & $\begin{array}{l}\text { R1 Do you hear when } \\
\text { someone ring the bell } \\
\text { or nock on the door? }\end{array}$ & $95 \%$ & PA & IN & IN \\
\hline & $\begin{array}{l}\text { T2 Você escuta quando } \\
\text { alguém toca a } \\
\text { campainha ou bate na } \\
\text { porta? }\end{array}$ & $\begin{array}{l}\text { R2 Do you listen when } \\
\text { anybody ring the door } \\
\text { bell or does it knock } \\
\text { on the door? }\end{array}$ & $80 \%$ & PA & IN & IN \\
\hline \multirow[t]{2}{*}{$\begin{array}{l}\text { 15) Do people avoid you } \\
\text { because of your hearing } \\
\text { difficulties? }\end{array}$} & $\begin{array}{l}\text { T1 As pessoas lhe evitam } \\
\text { por causa da sua } \\
\text { dificuldade de ouvir? }\end{array}$ & $\begin{array}{l}\text { R1 Other people avoid you } \\
\text { because of your hearing } \\
\text { difficulty? }\end{array}$ & $100 \%$ & PA & IN & IN \\
\hline & $\begin{array}{l}\text { T2 As pessoas lhe evitam } \\
\text { por causa das suas } \\
\text { dificuldades auditivas? }\end{array}$ & $\begin{array}{l}\text { R2 Do the people avoid } \\
\text { you because of your } \\
\text { hearing difficulties? }\end{array}$ & $90 \%$ & IN & IN & IN \\
\hline \multirow[t]{2}{*}{$\begin{array}{l}\text { 16) At present, would } \\
\text { you say that you lack } \\
\text { self-confidence because } \\
\text { of your hearing } \\
\text { difficulty? }\end{array}$} & $\begin{array}{l}\text { T1 No presente, você } \\
\text { diria que você perdeu } \\
\text { a autoconfiança por } \\
\text { causa do seu problema } \\
\text { de audição? }\end{array}$ & $\begin{array}{l}\text { R1At the moment, would } \\
\text { you say that you have } \\
\text { lost the self-confident } \\
\text { because of the hearing } \\
\text { problem? }\end{array}$ & $95 \%$ & IN & IN & IN \\
\hline & $\begin{array}{l}\text { T2 Atualmente, você } \\
\text { acha que você está } \\
\text { menos seguro por } \\
\text { causa da sua } \\
\text { dificuldade auditiva? }\end{array}$ & $\begin{array}{l}\text { R2 N owadays, do you } \\
\text { think you are less safe } \\
\text { because of your hearing } \\
\text { difficulty? }\end{array}$ & $65 \%$ & M A & PA & PA \\
\hline \multirow[t]{2}{*}{$\begin{array}{l}\text { 17) Do you find that } \\
\text { although you can hear } \\
\text { someone speaking, you } \\
\text { cannot understand what } \\
\text { they're saying? }\end{array}$} & $\begin{array}{l}\text { T1 Você acha que embora } \\
\text { você consiga escutar } \\
\text { alguém falando você } \\
\text { não consegue } \\
\text { entender o que a } \\
\text { pessoa está dizendo? }\end{array}$ & $\begin{array}{l}\text { R1Do you think that even } \\
\text { if you could hear } \\
\text { someone talking you } \\
\text { don't understand what } \\
\text { the person is saying? }\end{array}$ & $85 \%$ & IN & IN & IN \\
\hline & $\begin{array}{l}\text { T2 Você acha que apesar } \\
\text { de escutar o que as } \\
\text { pessoas dizem, você } \\
\text { não consegue } \\
\text { entender o que elas } \\
\text { estão dizendo? }\end{array}$ & $\begin{array}{l}\text { R2 Do you think in spite } \\
\text { of listening what the } \\
\text { people say, you don't } \\
\text { get to understand what } \\
\text { they are saying? }\end{array}$ & $85 \%$ & IN & IN & IN \\
\hline
\end{tabular}




\begin{tabular}{|c|c|c|c|c|c|c|}
\hline \multirow[t]{2}{*}{ Formulário original } & \multirow[t]{2}{*}{ Traduções } & \multirow[t]{2}{*}{ Retraduções } & \multirow{2}{*}{$\begin{array}{l}\text { A1- } \\
\text { Significado } \\
\text { referencial }\end{array}$} & \multicolumn{3}{|c|}{ A2- Significado geral } \\
\hline & & & & Exam 1 & Exam 2 & Exam 3 \\
\hline \multirow[t]{2}{*}{$\begin{array}{l}\text { 18) Do you hear the } \\
\text { telephone ringing from } \\
\text { another room? }\end{array}$} & $\begin{array}{l}\text { T1 Você escuta o telefone } \\
\text { tocando se estiver em } \\
\text { outra sala? }\end{array}$ & $\begin{array}{l}\text { R1Do you hear the } \\
\text { telephone ringing if you } \\
\text { were at other room? }\end{array}$ & $100 \%$ & PA & IN & IN \\
\hline & $\begin{array}{c}\text { T2 Você consegue escutar } \\
\text { o telefone tocando se } \\
\text { estiver em outra sala? }\end{array}$ & $\begin{array}{l}\text { R2 Do you get to listen the } \\
\text { telephone ringing if it } \\
\text { is in another room? }\end{array}$ & $90 \%$ & PA & IN & IN \\
\hline \multirow[t]{2}{*}{$\begin{array}{l}\text { 19) Do you ever get the } \\
\text { feeling of being cut off } \\
\text { from things because of } \\
\text { your hearing difficulty? }\end{array}$} & $\begin{array}{l}\text { T1 Você tem o } \\
\text { sentimento de estar } \\
\text { sendo cortado das } \\
\text { coisas por causa da sua } \\
\text { dificuldade de ouvir? }\end{array}$ & $\begin{array}{l}\text { R1Do you feel that you } \\
\text { have been cut off from } \\
\text { situations because of } \\
\text { your hearing difficulty? }\end{array}$ & $85 \%$ & IN & IN & IN \\
\hline & $\begin{array}{l}\text { T2 Você já teve a } \\
\text { sensação de ser } \\
\text { isolado das situações } \\
\text { por causa da sua } \\
\text { dificuldade auditiva? }\end{array}$ & $\begin{array}{l}\text { R2Did you already have } \\
\text { the sensation of being } \\
\text { isolated of the } \\
\text { situations because of } \\
\text { your hearing difficulty? }\end{array}$ & $75 \%$ & PA & PA & IN \\
\hline \multirow[t]{2}{*}{$\begin{array}{l}\text { 20) Do you feel that your } \\
\text { hearing condition has an } \\
\text { influence on the } \\
\text { relationship you have } \\
\text { with your spouse or a } \\
\text { person close to you? }\end{array}$} & $\begin{array}{l}\text { T1 Você sente que sua } \\
\text { condição auditiva tem } \\
\text { uma influência no } \\
\text { relacionamento com } \\
\text { seu(sua) } \\
\text { companheiro(a) ou } \\
\text { com alguma pessoa } \\
\text { próxima a você? }\end{array}$ & $\begin{array}{l}\text { R1Do you feel that your } \\
\text { hear condition } \\
\text { interfere in the } \\
\text { relationship with your } \\
\text { partner or with } \\
\text { someone else close to } \\
\text { you? }\end{array}$ & $90 \%$ & IN & IN & IN \\
\hline & $\begin{array}{l}\text { T2 Você acha que a sua } \\
\text { condição auditiva tem } \\
\text { influência no seu } \\
\text { relacionamento com } \\
\text { seu(sua) } \\
\text { companheiro(a) ou } \\
\text { com alguma pessoa } \\
\text { próxima a você? }\end{array}$ & $\begin{array}{l}\text { R2Do you think your } \\
\text { hearing condition has } \\
\text { influence in your } \\
\text { relationship with your } \\
\text { wife/husband or with } \\
\text { some close person? }\end{array}$ & $100 \%$ & PA & IN & IN \\
\hline
\end{tabular}

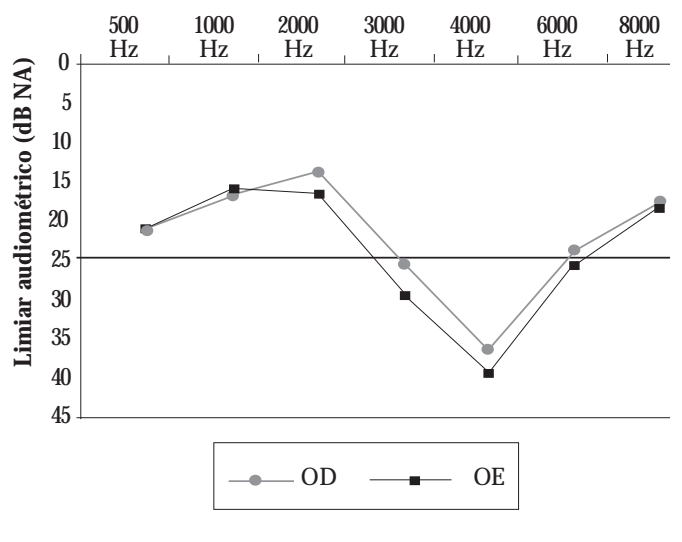

Gráfico 1. Limiares audiométricos médios dos trabalhadores portadores de PAIRO para as orelhas direita e esquerda.
$\mathrm{O}$ alfa de Cronbach estimado para os quatro fatores da versão em português do HDHS foram: 0,70 para o fator 1 (percepção da fala); 0,78 para o fator 2 (sons não verbais); e 0,56 para 0 fator 3 (sofrimento interpessoal); e 0,47 para 0 fator 4 (ameaça à autoimagem), todos apresentados na Tabela 3.

Esta tabela também apresenta a análise fatorial exploratória que foi realizada com os vinte itens da escala de inabilidades e handicap auditivo utilizando a análise de componente principal seguida pela rotação varimax. Uma solução de quatro fatores emergiu, com o auxílio do Cattel's scree test. Essa solução explicou $57 \%$ da variância total. 
Após a realização dessa análise fatorial, observou-se que especialmente dois itens tiveram correlações muito baixas com as suas dimensões: os itens 1 (dificuldade de acompanhar uma conversa normalmente) e 15 (as pessoas Ihe evitam por causa da sua dificuldade de ouvir).
A correlação item-total variou de 0,12 a 0,60 para este estudo. As médias de correlação itemtotal mais fortes foram encontradas entre os fatores $1(0,46)$ e $2(0,57)$ e as mais fracas foram para os fatores $3(0,38)$ e $4(0,22)$.

Tabela 3. Estrutura de fatores da Escala de Inabilidades e H andicap Auditivo, correlações item-total, autovalores, consistência interna e explicação da variância acumulada para cada fator.

\begin{tabular}{|c|c|c|c|}
\hline Fatores & $\begin{array}{l}\text { Correl ação } \\
\text { Item-Total }\end{array}$ & $\begin{array}{l}\text { Alfa de } \\
\text { Cronbach }\end{array}$ & $\begin{array}{l}\text { Explicação da variância } \\
\text { (cumulativa) }\end{array}$ \\
\hline F1 (percepção de fala) & & 0,70 & $19,0 \%$ \\
\hline $\begin{array}{l}\text { Pergunta } 1 \\
\quad \text { Dificuldade de acompanhar uma conversa }\end{array}$ & 0,12 & Autoval or $=3,6$ & \\
\hline $\begin{array}{l}\text { Pergunta } 5 \\
\quad \text { Dificuldade de ouvir TV }\end{array}$ & 0,56 & & \\
\hline $\begin{array}{l}\text { Pergunta } 9 \\
\quad \text { Dificuldade de ouvir rádio }\end{array}$ & 0,60 & & \\
\hline $\begin{array}{l}\text { Pergunta } 13 \\
\quad \text { Conversação em grupo }\end{array}$ & 0,51 & & \\
\hline $\begin{array}{l}\text { Pergunta } 17 \\
\text { Ouve, mas não entende }\end{array}$ & 0,53 & & \\
\hline F2 (sons não verbais) & & 0,78 & $34,1 \%$ \\
\hline $\begin{array}{l}\text { Pergunta } 2 \\
\quad \text { Porta abrindo }\end{array}$ & 0,54 & Autovalor $=2,9$ & \\
\hline $\begin{array}{l}\text { Pergunta } 6 \\
\text { Ouve água fervendo }\end{array}$ & 0,59 & & \\
\hline $\begin{array}{l}\text { Pergunta } 10 \\
\text { Ouve passos }\end{array}$ & 0,64 & & \\
\hline $\begin{array}{l}\text { Pergunta } 14 \\
\text { Soar da campainha }\end{array}$ & 0,59 & & \\
\hline $\begin{array}{l}\text { Pergunta } 18 \\
\text { Ouve o telefone tocar }\end{array}$ & 0,51 & & \\
\hline F3 (sofrimento interpessoal) & & 0,56 & $46,5 \%$ \\
\hline $\begin{array}{l}\text { Pergunta } 8 \\
\quad \text { Restrição à vida social }\end{array}$ & 0,31 & Autovalor $=2,3$ & \\
\hline $\begin{array}{l}\text { Pergunta } 12 \\
\text { Tensão e cansaço }\end{array}$ & 0,34 & & \\
\hline $\begin{array}{l}\text { Pergunta } 16 \\
\quad \text { Perdeu a autoconfiança }\end{array}$ & 0,32 & & \\
\hline $\begin{array}{l}\text { Pergunta } 19 \\
\quad \text { Sentimento de estar sendo cortado }\end{array}$ & 0,55 & & \\
\hline $\begin{array}{l}\text { Pergunta } 20 \\
\text { Influência no relacionamento com companheiro }\end{array}$ & 0,36 & & \\
\hline F4 (ameaça à autoimagem) & & 0,47 & $57,0 \%$ \\
\hline $\begin{array}{l}\text { Pergunta } 3 \\
\quad \text { Preocupação que as pessoas descubram o problema }\end{array}$ & na 0,17 & Autovalor $=2,0$ & \\
\hline $\begin{array}{l}\text { Pergunta } 4 \\
\quad \text { Difícil pedir que as pessoas repitam o que disseram }\end{array}$ & 0,17 & & \\
\hline $\begin{array}{l}\text { Pergunta } 7 \\
\quad \text { Fica contrariado se da uma resposta errada }\end{array}$ & 0,44 & & \\
\hline $\begin{array}{l}\text { Pergunta } 11 \\
\text { Incomodado ou chateado por ter dificuldade de } \\
\text { acompanhar uma conversa }\end{array}$ & 0,32 & & \\
\hline
\end{tabular}




\section{Discussão}

0 método de análise da equivalência semântica utilizada nesta pesquisa baseou-se no proposto por Reichenheim, M oraes e Hasselman ${ }^{23}$ e M oraes, Hasselmann eReichenheim ${ }^{24}$, queinclui cinco etapas consecutivas: (1) tradução do instrumento original, (2) retradução, (3) apreciação formal de equivalência, (4) pré-teste com amostra deconveniênciae (5) crítica final por especialistas na área com elaboração da versão final.

Observa-se que a disposição dos limiares auditivosno Gráfico 1 está compatível com a descrição da PAIRO segundo a Portaria $n 019$ do M inistério do Trabalho e Emprego ${ }^{25}$, que informa que a PAIRO é geralmente bilateral, não produz perdas maiores que $40 \mathrm{~dB} N \mathrm{~N}$ nas frequências baixas e que $75 \mathrm{~dB}$ NA nas altas, tem início e predomina nas frequências de 3000, 4000 ou $6000 \mathrm{~Hz}$, progredindo para 8000, 2000, 1000, 500 e $250 \mathrm{~Hz}$.

Uma particularidade em relação à análise da consistência interna dos itens deve ser levada em consideração: o tamanho da amostra. Prieto e Muñi ${ }^{26}$, ao citarem os padrões de avaliação de testes na Espanha, consideram que as amostras pequenas $(n<100)$ não são altamente recomendáveis para a avaliação dos dados psicométricos.

0 estudo de validação interna de um instrumento de qual idade de vida realizado por $\mathrm{M} \mathrm{CH}$ orney et al. ${ }^{27}$ utilizou o valor de 0,4 como ponto de corte para descarte de medidas. Já Pestana e Gagueiro ${ }^{28}$ consideram o índice de 0,6 como o mínimo aceitável para a confiabilidade do estudo.

$N$ ão há um ponto de corte ideal para a concepção de qualquer indicador; sendo assim, 0 nível de tolerância aceitável para o alfa de Cronbach deveser definido pelo investigador, levando em conta o valor obtido e a complexidade do fenômeno que se pretende medir $r^{29}$.

Foi considerado para este estudo o critério de Bowling ${ }^{30}$, que afirma que podem ser considerados como aceitáveis os valores do coeficiente alfa de Cronbach iguais ou superiores a 0,5.

Como a análise da consistência interna do questionário teve, neste estudo, um caráter exploratório, pode-se considerar que a Escala de Inabilidades e Handicap Auditivo exibiu, de forma geral, uma consistência interna aceitável para a maioria dos fatores, excetuando-se 0 fator 4 , com valor inferior a $0,5(0,47)$.

0 estudo de H étu et al. ${ }^{16}(n=232)$ identificou um coeficiente alfa no valor de 0,81 para o fator 1 (percepção de fala), 0,84 para o fator 2 (sons não verbais) e 0,84 para o fator 3 e 4 juntos (handicap). Já a pesquisa de Hallberg ${ }^{17}(n=101)$ en- controu os valores de alfa para os fatores $1,2,3$ e4, respectivamente de 0,$89 ; 0,85 ; 0,79$ e 0,84 .

A pesar de os valores de alfa de Cronbach terem sido menores que os encontrados no instrumento original em inglês, isso pode ter ocorrido devido ao número reduzido da amostra de trabal hadores participantes da pesquisa e ao fato de as perdas auditivas dos trabalhadores participantes não terem sido muito significativas, como demonstrado no audiograma médio dos trabaIhadores (Gráfico 1).

As qualidades psicométricas dos testes devem ser avaliadas constantemente; sendo assim, quanto mais informações sobre tais propriedades, maior será a credibilidade do teste ${ }^{31}$. Essa afirmação só reitera a importância de se realizar novos estudos com populações representativas de portadores de PAIRO para avaliar a adequação do instrumento proposto para a cultura brasileira.

0 item 15 obteve uma correlação muito baixa com os demais itens do fator $3(0,001)$, o que resultou na sua retirada da análise. Porém, vale salientar que, em pesquisas futuras, sugere- seque o item seja mantido para verificar se este fato se reproduz em amostras maiores e significativas da população para então ser pensada na sua exclusão da versão em português do HDHS.

O ptou-se por não retirar o item 1 da análise, apesar de ter tido uma correlação fraca $(0,12)$ quando comparado com os demais itens do fator 1 , já que a questão 1 (dificuldade de acompanhar uma conversa) relaciona-se a uma pergunta que provavelmente teria uma correlação maior caso a população apresentasse uma perda auditiva mais acentuada.

Em relação à população de estudo, em quase todos os casos, excetuando-se apenas um, os trabalhadores possuíam a área de fala (frequências de 500,1000 e $2000 \mathrm{~Hz}$ ) preservada, o queprovavelmentefez com queessa população não identificasse nenhuma dificuldade em acompanhar conversas.

As correlações dos itens para o fator 4 também se apresentaram fracas. M ais uma vez, uma possível justificativa para este fato seria o tamanho reduzido da amostra e as perdas auditivas que não eram tão acentuadas nesta população.

\section{Considerações finais}

A versão em português do instrumento H earing Disability and H andicap Scale (H DHS) para avaliação do handicap auditivo em portadores de PAIRO seguiu uma metodologia específica para 
adaptação transcultural de instrumentos e mostrou-se apropriada para aplicação na população de trabalhadores portadores de Perda Auditiva Induzida pelo Ruído Ocupacional.

$O$ instrumento foi considerado defácil compreensão e aplicação e obteve, de forma geral, confiabilidade e validade aceitáveis, considerando-se 0 tamanho reduzido da amostra e 0 fato de as perdas auditivas na população não terem sido muito acentuadas.

No entanto, a Escala de Inabilidades e $\mathrm{H}$ andicap Auditivo precisa ser testada em amostras maiores e representativas da população de trabalhadores brasileiros com PAIRO, a fim de ser ratificada a sua utilidade para a avaliação do handicap auditivo nessa população.

A disponibilização de um instrumento específico para portadores de PAIRO para o contexto brasileiro é de grande importância para incentivar e estimular a pesquisa nesse campo ainda pouco estudado no Brasil. Vale salientar que este instrumento pode ser utilizado ainda para populações expostas ao ruído em geral, no intuito de analisar as implicações psicossociais advindas dessa exposição.

Sendo assim, a versão em português do HDHS, denominada de Escala de Inabilidades e $\mathrm{H}$ andicap Auditivo, soma-se aos outros instrumentos de avaliação do handicap auditivo já existentes para possibilitar comparações de estudos realizados no Brasil com outros feitos em outros países.

A penas com a disseminação do conhecimento sobre o tema e com a adesão de novos pesquisadores que se poderá chegar a uma escala de handicap auditivo maisadequada, contribuindo principalmentepara o conhecimento edivulgação das implicações psicossociais advindas da PAIRO, a fim de conscientizar empregados e empregadores da importância de se evitar a instalação e progressão dessa doença silenciosa eirreversível.

\section{Colaboradores}

WTG Holanda e OB Coutinho-N eto participaram igualmente de todas as etapas da elaboração do artigo. 


\section{Referências}

1. Kwitko A. Coletânea 1: PAIR, PAIRO, RUÍDO, EPI, EPC, PCA, CAT, PERÍCIAS, REPARAÇÃO e outros tópicos sobre Audiologia Ocupacional. São Paulo: LTr; 2001.

2. Santino E, Couto HA. Audiometrias ocupacionais. Belo Horizonte: Ergo; 1995.

3. Ferreira Júnior M. PAIR: bom senso e consenso. São Paulo: VK; 1998.

4. Komniski TM, Watzlawick LF. Problemas causados pelo ruído no ambiente de trabalho. Revista Eletrônica Lato Sensu 2007; 2(1):147-160.

5. Gessinger R, Castoldi L, Fensterseifer LM. Efeitos psicossociais da perda auditiva induzida pelo ruído (PAIR). In: Nudelmann AA, Costa EA, Seligman J, I bañez RN, organizadores. PAIR: Perda Auditiva Induzida pelo Ruído. Porto Alegre: Bagaggem; 1997. p. 251-254.

6. Santos UP, M orata TC. Efeitos do ruído na audição. In: Santos UP, organizador. Ruído: riscos e pre venção. $2^{a}$ ed. São Paulo: Hucitec; 1996. p. 43-53.

7. World Health Organization. International classification of impairments, disabilities and handicaps: a manual of classifications relating to consequences of disease. Genebra: WHO; 1980

8. Stephens D, H ètu R. Impairment, disability and handicap in audiology: towards a consensus. Audiology 1991; 30(4):185-200.

9. Weinstein BE, Ventry I. Audiologic correlates of hearing handicap in the elderly. J Speech Hear Res 1983; 26(1):148-151.

10. Almeida K. Avaliação objetiva e subjetiva do benefício das próteses auditivas em adultos [tese]. São Paulo (SP): Escola Paulista de M edicina, Universidade Federal de São Paulo; 1998.

11. Corrêa GL, Russo ICP. Auto percepção do handicap em deficientes auditivos adultos e idosos. Revista CEFAC 1999; 1(1):54-63.

12. Bess FH, Williams AH, Lichtenstein MJ. Avaliação audiológica dos idosos. In: Musiek FE, Rintelmann $W F$, organizadores. Perspectivas atuais em avaliação auditiva. São Paulo: Manole; 2001. p. 343-369.

13. Noble WG, Atherley GRC.The Hearing M easurement Scale: a questionnaire for the assessment of auditory disability. Journal of Audiology Research 1970; 16:51-58.

14. High WS, Fairbanks G, Glorig A. Scale for self assessment of hearing handicap. J Speech Hear Disord 1964; 29:215-230.

15. Hallberg LRM, Johnsson T, Axelsson A. Structure of perceived handicap in middle-aged males with noise induced hearing loss, with and without tinnitus. Audiology 1993; 32:137-152.

16. Hétu R, Getty L, Philibert L, Noble WG, Stephens $D$. Development of a clinical tool for the measurement of the severity of hearing disabilities and handicaps. J Speech Lang Pathol Audiol 1994; 18:83-95.

17. Hallberg LRM. Evaluation of a Swedish version of the Hearing Disabilities and $\mathrm{H}$ andicaps Scale, based on a clinical sample of 101 men with noise-induced hearing loss. Scandinavian Audiology 1998; 27:23-29.

18. Barrenäs $M L$, Holgers KM. A clinical evaluation of the hearing disability and handicap scale in men with noise induced hearing loss. N oise \& $\mathrm{H}$ ealth 2000; 6:67-78.
19. Wieselberg M B. A auto avaliação do handicap em indivíduos idosos portadores de deficiência auditiva: o uso do HHIE [dissertação]. São Paulo (SP): Pontifícia Universidade Católica de São Paulo; 1997.

20. Reichenheim ME, Moraes $C L$. Alguns pilares para a apreciação da validade de estudos epidemiológicos. Rev. bras. epidemiol. 1998; 1:131-148.

21. M attos $P$, Serra-Pinheiro MA, Rohde LA, Pinto D. Apresentação de uma versão em português para uso no Brasil do instrumento MTA-SNAP-IV de avaliação de sintomas de transtorno do déficit de atenção/hiperatividade e sintomas de transtorno desafiador e de oposição. Rev. psiquiatr. Rio Gd. Sul 2006; 28(3):290-297.

22. Lima M OP. Qualidade de vida relacionada à saúde de mulheres grávidas com baixo nível de socioeconômico [dissertação]. São Paulo (SP): Universidade de São Paulo; 2006.

23. Reichenheim ME, Moraes $\mathrm{CL}, \mathrm{H}$ asselmann $\mathrm{MH}$. Equivalência semântica da versão em português do instrumento Abuse Assessment Screen para rastrear a violência contra a mulher grávida. Rev. Saude Publica 2000; 34(6):610-616.

24. Moraes $C L, H$ asselmann $M H$, Reichenheim ME. Adaptação transcultural para o português do instrumento "Revised conflict tactics Scales (CTS2)" utilizado para identificar violência entre casais. Cad Saude Publica 2002; 18(1):163-176.

25. Brasil. Ministério do Trabalho. Portaria no 19. [acessado 2004 fev 22]. Disponível em: http:// www.mtb.gov.br/Temas/SegSau/Legislacao/Portarias/ 1998/conteudo/port19.asp

26. Prieto G, Muñiz J. Un modelo para evaluar la calidad de los tests utilizados en España. [acessado $2008 \mathrm{fev}$ 24]. Disponível em: http://www.cop.es/vernumero. asp?id=41

27. M chorney CA, M CH orney CA, Ware JE Jr, Lu JF, Sherbourne CD. The M OS 36-item short-form health survey (SF-36): tests of data quality, scaling assumption, and reliability across diverse patient groups. M edical Care 1994; 32(1):40-66.

28. Pestana M H, Gagueiro JN. Análise de dados para Ciências Sociais. 4ạ ed. Lisboa: Síbalo; 2005.

29. Pereira JCR. Análise de dados qualitativos: estraté gias metodológicas para as ciências da saúde, humanas e sociais. São Paulo: Edusp; 1999.

30. Bowling A. M easuring health: a review of quality of life measurement scales. Philadelphia: Open University Press; 1997.

31. Anastasi A, Urbina S. Testagem psicológica. 7 a ed. Porto Alegre: Artes M édicas; 2000.

Artigo apresentado em 30/10/2008

Aprovado em 27/07/2009

Versão final apresentada em 28/09/2009 